\section{Resumen}

La relación entre educación e inclusión en el contexto andino puede analizarse considerando 4 desafíos. El primero, acceso a la educación, ha tenido una mayor visibilidad en las acciones de los gobiernos y ha supuesto en general avances destacados en la extensión de la cobertura en educación inicial y en secundaria, además de la práctica universalización de la primaria. El segundo, acceso equitativo a condiciones materiales básicas para el aprendizaje, ha estado presente a través de programas de atención integral, que han venido aportando alimentación, salud, vínculos comunitarios y familiares e incentivos económicos a los niños más pobres, en especial a la primera infancia. El tercero, acceso a logros educativos de calidad, está más relacionado al currículo y presenta mayores dificultades: rendimientos escolares bajos, especialmente en zonas rurales; brechas educativas que reflejan desigualdades sociales; deficiencias y limitaciones en las capacidades pedagógicas de los docentes, que se traducen en malos desempeños aún contando con currículos innovadores. Finalmente, el cuarto, la inclusión educativa como reflejo de la inclusión social y cultural de todos los grupos sociales, es el que presenta mayores problemas y desafíos: problemas de convivencia, integración y cohesión social en las instituciones escolares, de ciudadanía y de no reconocimiento del otro como sujeto con iguales derechos. Si bien este problema es expresión de un contexto social inequitativo, surge la pregunta por las posibilidades y responsabilidades que la educación, escuelas y educadores podemos y debemos asumir.

\section{Palabras clave}

Inclusión educativa, Región Andina, políticas educativas, prácticas pedagógicas, currículo

\section{Abstract}

The relationship between education and inclusion in the Andean context can be analyzed considering 4 challenges. The first, access to education, has had greater visibility in the actions of governments and in general, has been transformed into leading advances in the extension of coverage in early and secondary education, in addition to the practical universalization of primary education. The second, equitable access to basic material conditions for learning has been present through comprehensive care programs, which have been providing food, health, family and community ties and economic incentives for the poorest children, especially the first childhood. The third, access to quality educational achievements, is more related to the curriculum and presents major difficulties: low school performance, especially in rural areas; educational gaps that reflect social inequalities; shortcomings and limitations in teaching capacities, which translates into poor performances even with innovative curricula. The fourth, educational inclusion as a reflection of social inclusion and cultural development of all social groups is the one that presents greater problems and challenges: problems of coexistence, integration and social cohesion in schools, citizenship and non-recognition of the other as an individual with equal rights. While this problem is the expression of an inequitable social context, the question arises as to the possibilities and responsibilities that education, schools and educators can and should assume.

\section{Key words}

Inclusive education, Andean Region, educational policies, pedagogical practices, curriculum 


\title{
Educación e inclusión en la Región Andina'
}

\author{
Verónica Villarán Bedoya ${ }^{2}$ \\ Luis Guerrero Ortiz ${ }^{3}$
}

\section{Introducción}

En septiembre de 2007 se realizó el Taller Internacional sobre Inclusión Educativa en América Latina, que congregó a los representantes de las Comunidades de Práctica en Desarrollo Curricular de las regiones Andina y Cono Sur, en la sede de la Universidad de San Andrés, Buenos Aires. La actividad fue organizada por la Oficina Internacional de Educación (OIE-UNESCO), la División para la promoción de la Educación Básica (ED/BAS, UNESCO París), la Oficina Regional de Educación para América Latina y el Caribe (OREALC), el Ministerio de Educación de Argentina y la Escuela de Educación de la Universidad de San Andrés. El objetivo del encuentro fue discutir y empezar a construir acuerdos sobre los niveles de inclusión y exclusión educativa de los países latinoamericanos, los progresos alcanzados y el rol del currículo en esos esfuerzos, tomando como base los informes nacionales presentados

\footnotetext{
${ }^{1}$ Síntesis del Estudio Regional "Currículo e inclusión en el nivel inicial y el primer ciclo de la primaria: Visiones, estrategias, experiencias y buenas prácticas en la Región Andina" realizado por los investigadores Verónica Villarán Bedoya y Luis Guerrero Ortiz. Se enmarca en las actividades realizadas por la Comunidad Andina de Práctica en Desarrollo Curricular coordinada por la Oficina Internacional de Educación, OIE, de la UNESCO. El documento fue presentado en el Taller Internacional sobre Inclusión Educativa-Regiones Cono Sur y Andina (Buenos Aires, Argentina, 12 al 14 de septiembre de 2007) que constituyó una actividad preparatoria de la 48.a reunión de la Conferencia Internacional de Educación "Educación Inclusiva: El camino hacia el futuro" (25 al 28 de noviembre de 2008, Ginebra, Suiza, consultar www.ibe.unesco.org/International/ICE48/English/index.html). Los derechos de autor de la Síntesis del estudio regional pertenecen a la OIE-UNESCO. Texto recibido el 29 de febrero, evaluado el 19 y el 22 de mayo, y arbitrado el 9 de junio de 2008.

${ }^{2}$ Magíster en Ciencias Sociales con orientación en Educación, por la FLACSO, Argentina. Investigadora y formadora de la Facultad de Educación de la Universidad Peruana Cayetano Heredia. Coordinadora de la Comunidad Andina de Práctica en Desarrollo Curricular. veronicavb@yahoo.com

${ }^{3}$ Magíster en Política Educativa de la Universidad Alberto Hurtado, Chile. Asesor del Consejo Nacional de Educación en temas de Políticas Educativas. Miembro fundador de la Asociación Foro Educativo e integrante de su Consejo Directivo. Miembro de la Comunidad Andina de Práctica en Desarrollo Curricular. guerrerortiz@gmail.com
} 
por los países Andinos y del Cono Sur; y como referencia las presentaciones de otras Comunidades de Práctica de otras regiones del mundo. El presente documento es una síntesis del Informe Regional presentado en dicho Taller, el cual a su vez se basó en los informes nacionales de cinco países andinos: Bolivia, Colombia, Ecuador, Perú y Venezuela 4 .

\section{Marco conceptual}

La inclusión ha sido un concepto comúnmente restringido a alumnos con necesidades educativas especiales, sobre todo a quienes mostraban algún tipo de discapacidad. La manera de afrontarla hasta la década pasada, ha sido a través de medidas compensatorias o remediales, en contextos separados de aquellos donde se educaban los estudiantes normales. Según la OIE de la UNESCO (2007), la Conferencia Mundial de Salamanca en 1994 marca un hito en el cambio de esta concepción, redefiniendo la educación inclusiva como integración de todo niño excluido, discriminado, segregado por cualquier condición personal, física, cultural o social.

De entonces a la fecha se han venido ensayando en diversos lugares del planeta distintos modelos de inclusión, empezando por la matrícula de todos los niños en las escuelas comunes, pero sin avanzar suficientemente en la adecuación de los currículos y la pedagogía a la diversidad del aula. Este desajuste fue haciendo patente la necesidad de avanzar de la inclusión como acceso a oportunidades para recibir educación, insuficiente por sí sola, a la inclusión como acceso a oportunidades de aprendizaje de equivalente calidad, atendiendo las diferencias. De lo contrario, el sistema terminaba por excluir a los diferentes; es decir, a los que se diferenciaban del prototipo ideal de usuario: un alumno afín al código universal de la cultura escolar y con todas las aptitudes y prerrequisitos necesarios para aprender bajo esos parámetros.

Emilio Tenti (2007) sostiene, en este mismo sentido, que una de las dimensiones de la exclusión educativa tiene que ver con estar fuera de la escuela, pero la otra tiene que ver con la exclusión del conocimiento. A la incorporación de los excluidos, sobre todo niños menores de 5 años y adolescentes, se suma ahora la exigencia de desarrollar en las personas un conjunto de saberes y valores indispensables para su autonomía e inserción en la sociedad de hoy. Tenti dirá, sin embargo, que

La exclusión del conocimiento es un fenómeno más complejo de la exclusión escolar. En ambos casos el problema no se resuelve únicamente expandiendo

\footnotetext{
${ }^{4}$ El Informe Regional fue elaborado por Luis Guerrero, tomando como base los informes nacionales de Bolivia (Crista Wiese), Ecuador (Carlos Jiménez), Colombia (Gloria Clavo), Perú (Luis Guerrero y Karin Rivas) y Venezuela (Carlota Terán). Estos informes están disponibles y pueden consultarse para ampliar la información que aquí se reseña, previa solicitud escrita al correo electrónico de Verónica Villarán Bedoya. veronicavb@yahoo.com
} 
la oferta escolar, como lo hacía la política educativa tradicional. La escolarización y con mayor razón el desarrollo de conocimientos supone una demanda real por parte de los aprendices y sus familias. Pero esta demanda de escolarización y conocimiento no puede darse por descontada, sino que es el resultado de condiciones sociales determinadas. El aprendizaje tiene lugar si existe una demanda y se dan determinadas condiciones escolares y pedagógicas adecuadas a las condiciones y características de los aprendices, que son cada vez más desiguales y diferentes (p. 1).

Esta doble dimensión de la exclusión es también un doble desafío de inclusión para la educación, que implica ahora al conjunto de la sociedad no sólo profundamente diversa, sino además, dividida y desigual.

La inclusión, según reciente definición de la UNESCO (2005), es

el proceso de identificar y responder a la diversidad de las necesidades de todos los estudiantes a través de la mayor participación en el aprendizaje, las culturas y las comunidades, y reduciendo la exclusión en la educación. Involucra cambios y modificaciones en contenidos, enfoques, estructuras y estrategias, con una visión común que incluye a todos los niño/as del rango de edad apropiado y la convicción que es la responsabilidad del sistema regular, educar a todos los niño/as (p. 13).

Si consideramos que nuestros sistemas educativos han sido excluyentes y uniformizadores por tradición, se entenderá fácilmente que su adecuación a la diversidad sociocultural, a las diferencias individuales y a las desigualdades sociales que caracterizan nuestras sociedades, son retos de gran envergadura para las políticas educativas, para las instituciones escolares y para los docentes.

Como dice Aguerrondo (2007), los sistemas educativos fueron siempre selectivos, por lo que la noción de inclusión aparece como una ventana de oportunidad porque hace evidente la necesidad de constituir otro sistema educativo, diseñado para cumplir una función diferente. En momentos en que la globalización está generando exclusión como parte de su desarrollo, "la idea de 'incluir' debe ser una idea-fuerza que se sostenga desde la necesidad de una sociedad más justa y democrática” (p. 3). Así, la inclusión educativa implica la posibilidad de todas las personas a ejercer su derecho a la educación y, por lo tanto, a adquirir las capacidades que hagan posible una participación social y política conciente e informada, así como efectivo su derecho a la igualdad de oportunidades. En ese sentido se asocia a la posibilidad de poder constituir sociedades multiculturales y democráticas, integradas y sin minorías discriminadas.

Desde esta perspectiva, Aguerrondo afirma que la inclusión no es una nueva meta, sino la base de un nuevo paradigma educativo: 
La unidad de cambio ya no es el alumno, ni el aula, ni la escuela, sino el sistema educativo como tal, lo que implica por supuesto cambiar (cómo son) las escuelas y (quiénes son) los alumnos. Pero también implica proponer otro currículo, otra propuesta de enseñanza, otra organización de las escuelas, otra formación docente, otro perfil de quien debe ser docente, otra distribución territorial y espacial de las escuelas, otros sistemas de control de calidad, y así por demás $(2007$, p. 3).

Así, la construcción de currículos inclusivos implica necesariamente la redefinición de la pedagogía, de la docencia y de la institucionalidad escolar, así como de la estructura y gestión de los sistemas educativos. No será una sola variable la que haga posible la inclusión.

\section{Educación e inclusión en los países andinos ${ }^{5}$}

\section{Visiones educativas, sociales y políticas respecto a la inclusión educativa}

El tema de la inclusión educativa en Bolivia es aún poco trabajado. Se adhiere oficialmente a los referentes internacionales, en los que se asocian la inclusión social y educativa, enfatizándola como una postura ética frente a la desigualdad e injusticia social y como un esfuerzo por favorecer las condiciones para el ejercicio de derechos y de una ciudadanía plena. Se asume la exclusión social como expresión de condiciones económicas, sociales y culturales que la educación puede contribuir a revertir, otorgando especial atención a poblaciones en desventaja por desplazamientos migratorios, origen y riesgo social o discapacidades. Por lo tanto, se postula que las políticas de inclusión social deben tener un fuerte componente educativo y mejorar las condiciones de equidad y justicia social.

No se ha construido una postura consensuada en instituciones privadas y públicas, habiendo múltiples sentidos y propuestas sobre esta noción, lo que no permite una visión común de los desafíos, ni afrontarlo de manera sistemática. No obstante, la concepción predominante de inclusión educativa la remite fuertemente a la educación especial y a la integración escolar. En este grupo se ubican una gran parte de las iniciativas y constituye una de las visiones más fuertes, orientando las acciones

\footnotetext{
${ }^{5}$ Datos de contexto económico, social y educativo por cada país pueden ser consultados en los informes nacionales, solicitando los mismos al correo electrónico referido anteriormente. Basta señalar acá que en general los países andinos tienen una población mayoritariamente joven, con altos índices de pobreza y desempleo. Los niños y niñas van en su mayoría a la escuela pública, con índices cercanos a la universalidad en primaria, pero en menor proporción en la escuela inicial y en la secundaria. Asimismo, se presentan grandes disparidades en cobertura y calidad en escuelas rurales con respecto a las urbanas; y en general, la región tiene bajos índices de calidad educativa según evaluaciones nacionales e internacionales realizadas en los últimos 10 años.
} 
inclusivas únicamente a la integración de niños especiales en la enseñanza regular. En este grupo encontramos muchas instituciones de iniciativa privada o vinculadas a iglesias.

De todos modos, de manera creciente se asocia la inclusión educativa con factores de orden social y cultural, con las diferencias de carácter socioeconómico, las étnicas-culturales, las de género y las de capacidades especiales como factores de exclusión, aunque poniendo distinto énfasis en cada uno y sin lograr una visión integral de la inclusión educativa. El énfasis en las diferencias étnicas y culturales es una visión que aparece en la reforma educativa de 1994 y es el acento principal en las leyes y reformas emprendidas, en las políticas y prácticas curriculares de interculturalidad y de bilingüismo. El énfasis en los factores sociales y el género se encuentran iniciativas más bien asistenciales o remediales, que buscan la ampliación de la cobertura, la permanencia a través de bonos, la asistencia integral alimentaria y la atención en salud a la primera infancia, a través de programas de carácter estatal, que derivan de políticas de reducción de la pobreza con poca relación con las políticas educativas. Algunos de ellos trabajan desde la especificidad de género, promoviendo la inclusión y permanencia de las niñas en la escuela rural.

Todas las conceptualizaciones encontradas hacen referencia a acciones orientadas a lograr un acceso equitativo a la educación, superando las diferencias discriminatorias, aunque es menos evidente encontrar el sentido de la educación inclusiva como un factor para romper las condiciones de desigualdad social.

En Colombia, la inclusión, tal como se propone a nivel oficial, se entiende como la atención preferencial a los desplazados por la violencia, a la población indígena, a los niños discapacitados y a la población de las áreas rurales de baja densidad, mediante proyectos educativos y la atención ágil a la población desplazada, acompañada por programas de retorno. Se cuenta con modalidades especiales para la inclusión educativa de los grupos indígenas, a la vez que se continúa con la política de ampliación de atención especializada para niños, niñas y jóvenes con limitaciones o discapacidades.

Así, la inclusión se concibe como el mecanismo para evitar cualquier tipo de discriminación, gracias al cambio progresivo de concepciones y prácticas excluyentes. Es por eso que otro elemento importante asociado a la inclusión es el fomento de la cultura de la diversidad, lo que implica entre otras cosas la inclusión de personas con necesidades educativas especiales, tanto a nivel del desempeño en el aprendizaje, como en la oferta de servicios educativos y sociales.

Esta visión de la inclusión es la que hace que los proyectos del gobierno nacional estén focalizados en la atención preferencial de la población desplazada por la violencia, la población indígena y afro colombiana, los niños discapacitados y la población de las áreas rurales de baja densidad.

En Ecuador, la inclusión educativa es un tema relativamente nuevo que nace desde la Educación Especial tanto pública como privada. En los 90, con la adhesión 
del país a los acuerdos internacionales basados en los principios de la Educación para Todos (EPT), se resalta el derecho de todas las personas a la educación, independientemente sus condiciones físicas, intelectuales, sociales, emocionales, lingüísticas u otras. Así, inclusión empieza a aludir a niños discapacitados y niños bien dotados, a niños que viven en la calle y que trabajan, niños de poblaciones remotas o nómadas, niños de minorías lingüísticas étnicas o culturales, y niños de otros grupos o zonas, desfavorecidos o marginados. En los últimos años se habla fuertemente de inclusión como el hecho de incluir a los excluidos de los grupos indígenas, no como una iniciativa del Estado, sino como producto de una lucha de los propios movimientos.

Pese a existir un sinnúmero de visiones sobre la inclusión, se percibe interés por avanzar en este terreno. Hay nuevas políticas inclusivas que empiezan a surgir, por ejemplo, en el recién creado Ministerio de Inclusión Social y Económica. El proceso de transformación política y económica que se está produciendo en el país es una oportunidad para construir una agenda nacional inclusiva, por ejemplo, a través de la Asamblea Constituyente.

El concepto de inclusión educativa en el Perú se ha venido relacionando comúnmente en el ambiente educativo con la inserción de niños con discapacidad en instituciones de educación básica regular, pero entre los expertos hay coincidencia en que la inclusión se vincula con ofrecer las mismas oportunidades de desarrollo a todos los grupos excluidos, sea por pobreza, etnia, ruralidad, género, discapacidad, etc. Se asume que la discriminación constituye una característica estructural del sistema educativo, que tiende a excluir por razones de rendimiento, muchas veces asociadas a aspectos étnicos, expresiones lingüísticas, género o, simplemente, pobreza. También se asume que la exclusión no es un problema exclusivo de la educación, sino que proviene del mundo social, donde el fenómeno se constata en todos los escenarios.

Todos coinciden en que en la última década, la educación se ha vuelto más inclusiva. Pero los logros en este aspecto enfatizan sobre todo la cobertura y la normatividad. Pero la inclusión en el ámbito del currículo implica otras complejidades. Desde el punto de vista del currículo normado, inclusión es no segregación, apertura a lo diverso, pluralidad cultural; pero en el plano del currículo implementado, debiera ser equidad en las condiciones de aprendizaje y en los resultados. Equidad e inclusión en educación son conceptos recurrentes, en la medida que suponen acceso igualitario y no exclusión en ningún terreno. Por eso se piensa que la inclusividad del currículo debiera estar asociada no sólo a sus contenidos sino también a las condiciones de su desarrollo.

El currículo puede, normativamente, estar dirigido a los niños de todos los grupos sociales, y formulado con amplitud; pero las expectativas de los docentes hacia sus estudiantes suelen estar teñidas de prejuicio. La exclusión en sus diversas expresiones y motivos suele estar asociada a la pobreza, pero también al prejuicio y 
a los malos pronósticos de los que suele ser objeto en el mundo escolar. Pero todos coinciden en la necesidad de llegar a consensos más claros sobre las distintas significaciones que se otorga al concepto de inclusión, tanto en el ámbito del currículo normado como del currículo vivido. El tema es aún ambiguo y se requieren acuerdos que deriven en políticas ex profesas de inclusividad curricular.

En el caso de Venezuela, la idea de inclusión del Estado pone mucho énfasis en la cobertura y busca por eso incrementar tanto el acceso como la permanencia en el sistema educativo, propiciando una igualdad de oportunidades en cuanto al aprendizaje integral y de calidad. Las políticas de inclusión educativa y social están focalizadas en los niños entre 0 y 6 años de edad, la población rural e indígenas y de fronteras y los niños y jóvenes excluidos del sistema educativo formal. El objetivo central es, pues, superar la exclusión educativa que han sufrido durante años.

En el marco de su política de inclusión, orientada a compensar deficiencias en la cobertura de la educación básica a nivel de niños y jóvenes mayores de 15 años de edad, el Estado desarrolla programas para la inclusión de quienes han estado fuera del sistema de educación formal, tanto en la básica como en la media y superior, ofreciéndole vías alternativas no formales (Misiones). En esa misma perspectiva, la Educación Bolivariana busca neutralizar la baja en la tasa de matrícula escolar de los últimos años, intensificando programas alimentarios que contrarresten variables que influyen en la deserción, repitencia y baja prosecución de la Educación básica; la atención de minorías nacionales autóctonas excluidas de las políticas públicas, respetando su idiosincrasia cultural. Se focalizan esfuerzos particularmente en los grupos indígenas, incorporando su lengua y sus costumbres a las acciones educativas de la escuela.

\section{Estrategias educativas para el logro de la inclusión educativa}

\section{Implementación de políticas en torno a un currículo inclusivo}

En Bolivia, las estrategias inclusivas desarrolladas en educación inicial, en relación al ámbito estrictamente curricular, han pasado por el propio diseño del currículo, flexible, abierto, integrado y global, que toma en cuenta el juego como principio base de la expresión, la exploración, la construcción de conocimientos, y que promueve el desarrollo integral de las capacidades del niño, buscando establecer una relación estrecha con la familia y la cultura; y han comprendido así mismo el trabajo con proyectos de aula, orientado al desarrollo de competencias, la resolución de problemas reales y el acceso a aprendizajes significativos. En la educación primaria, también se ha tomado en cuenta el juego como principio base de la expresión, exploración y construcción de conocimientos, asumiendo que el niño construye conocimientos desde la complejidad de la realidad que lo rodea. Al igual que en el nivel de inicial, propone establecer una relación estrecha de la escuela con la familia y la cultura; establece el uso de rincones de aprendizaje y de módulos didácticos, con 
contenidos y actividades. Si bien existe un alto nivel de prescripción del currículo, se prevé que cada unidad educativa haga las adaptaciones curriculares necesarias considerando las características socioculturales de cada región.

En el caso de Colombia, para mejorar la pertinencia y la calidad del servicio educativo a las poblaciones vulnerables y combatir la deserción, se han implementado modelos educativos flexibles, acompañados de nuevas herramientas pedagógicas y didácticas, canastas educativas y formación de funcionarios, directivos docentes y docentes. Entre 2003 y 2006, se capacitaron 34.781 docentes en modelos flexibles y en atención a poblaciones vulnerables y se implementaron 15.087 experiencias de modelos educativos flexibles.

En Ecuador, el currículo para la educación básica tiene aspectos que favorecen procesos educativos inclusivos. Los maestros de la escuela regular que trabajan con estudiantes con alguna discapacidad y las instituciones de educación especial llevan el mismo currículo, aún cuando no se tiene evidencia de que se efectúen las adaptaciones necesarias en el primer caso. En realidad, se carece de recursos para una adecuada implementación, hay carencia de medios y tecnología, no se permite modalidades alternativas de capacitación, hay una disociación entre gestión administrativa y gestión pedagógica, falta concreción en el trabajo en equipo, se prioriza la enseñanza sobre el aprendizaje, se pide promover la enseñanza activa y participativa, el aprendizaje significativo y cooperativo, el desarrollo del pensamiento critico y la creatividad, cuando el proceso de formación del profesorado contradice estos principios y no se le prepara para enseñar de esta manera.

En el caso de Perú, todos coinciden en que si bien el Diseño Curricular Nacional (DCN) demanda aprendizajes que hacen referencia a la educación en ciudadanía, la interculturalidad o la diversificación de sus contenidos, conceptos que posibilitan la inclusividad y sirven de horizonte al trabajo pedagógico, el problema social de la exclusión-inclusión se menciona de manera muy general. Hay quienes piensan que la inclusividad está en el concepto de diversificación curricular, pero para los docentes la diversificación curricular es sólo una adecuación nominal. Una implementación inclusiva del currículo requiere capacitación de maestros, acompañamiento, contratación y nombramiento de maestros con oportunidad, materiales a disposición de todos, normas que apoyen el proceso en lugar de frenarlo o desviarlo, difusión de los cambios a los padres de familia, a la comunidad y a los medios, evaluación pertinente seguida de medidas correctivas oportunas, infraestructura, equipamiento, etc. Exige así mismo que el currículo se articule con esta gran necesidad nacional de cohesión e integración social. Pese a hacer mención a la necesidad de promover aprendizajes tomando en cuenta la diversidad sociocultural e individual del aula, la norma no tiene correlato en los hechos pues se sigue capacitando para una enseñanza estandarizada y uniforme. La pedagogía que predomina no es inclusiva, la implementación de los cambios curriculares no ha logrado convencer y transformar cualitativamente la práctica docente. 
En el caso de Venezuela, el modelo Simoncito constituye una estrategia efectiva para asegurar el acceso a la educación inicial de todos los niños, en particular de los sectores más desfavorecidos del país, en igualdad de condiciones, asegurándoles la alimentación, la salud y compensación a sus desventajas iniciales. En él se atienden a los niños en la etapa maternal ( 0 a 3 años) y en la preescolar ( 3 a 6 años o hasta su ingreso al primer grado). En relación con la educación básica en general, se desarrollan acciones con la familia de carácter intersectorial y con instituciones del área de las ciencias, las artes y el deporte. Además, todos los miembros de la comunidad educativa, incluyendo los alumnos, elaboran el Proyecto pedagógico de aula. La meta planteada era la universalización de la educación bolivariana en el país para antes del año 2006.

\section{Docentes: áreas desarrolladas y competencias personales}

En Bolivia se dio un fuerte impulso a la formación de profesores en Educación Intercultural Bilingüe. Así mismo, se han diseñado los currículos de formación de profesores para educación inicial y primaria con un enfoque flexible que rescata la diversidad cultural. En relación con la atención que debe brindar la escuela regular a los alumnos con necesidades educativas especiales se ha avanzado en la formación de maestros del nivel inicial y primario en el área de integración educativa. Si bien se ha avanzado a nivel de la normativa y de las propuestas curriculares, los procesos de cambio curricular no están propiciando la generación de prácticas inclusivas en el aula, esto no se concreta en prácticas reales. Las escuelas no han logrado propiciar espacios de inclusión y considerar la diversidad de habilidades y capacidades de los estudiantes. Existen incontables resistencias para el trabajo con adaptaciones curriculares y la inclusión educativa es aún un conjunto de buenas intenciones poco comprendidas por los profesores. La comprensión de la necesidad y de la posibilidad de inclusión de alumnos con capacidades y habilidades diferentes, es un supuesto negado que las políticas prefieren ignorar.

En el caso de Colombia, el Ministerio forma a los maestros en temáticas relacionadas con herramientas pedagógicas y desarrollo de competencias para la convivencia. Los talleres de herramientas pedagógicas se realizan por ciclos que comprenden aspectos como derechos humanos y de los niños; cultura de paz, sobre todo en un contexto de desplazamiento; salud sexual y reproductiva, a fin de hacerles conocer a los estudiantes sus derechos sexuales y reproductivos; herramientas pedagógicas para identificar necesidades en el caso de niños y jóvenes afectados por la violencia; competencias ciudadanas, en especial la capacidad para interactuar con su ambiente; competencias personales, que incluyen aspectos motivacionales, afectivos $\mathrm{y}$ actitudinales.

En Ecuador existen 114.350 docentes en el sector educativo estatal y 69.541 docentes particulares. Pese a ser un país sumamente diverso, ninguno de estos docentes ha sido formado para la atención a la diversidad, ni en las Facultades, las 
Escuelas de Ciencias de la Educación, ni en los Institutos Pedagógicos de Formación de Maestros. Su formación inicial, además, aún no se adecua coherentemente a la propuesta de la Reforma. Por añadidura, para muchos docentes la reforma curricular ha sido sólo una simple novelería que se ha limitado a cambiar un término por otro.

En el caso de Perú, el DCN norma que se implemente en las aulas la atención adecuada a la diversidad, pero no se han creado espacios para construir con los docentes acuerdos sobre el significado de los nuevos aprendizajes demandados y sus enormes implicancias para la enseñanza. En realidad, el conjunto de discriminaciones por desigualdades (de género, etnia, cultura, desigualdad social) opera de manera entremezclada en el ámbito social y es esto lo que se expresa también en el plano educativo. En ese sentido, todos coinciden en la necesidad de desarrollar capacidades en el docente para la educación en ciudadanía y la convivencia, para el fortalecimiento de la dimensión personal y para afrontar el reto de la inclusión y la diversidad. El problema es que esto no es sólo ni principalmente una cuestión de metodologías, sino de la calidad de las interacciones con los estudiantes, lo que implica actualizar conocimientos, revisar actitudes, saber qué y cómo se trabaja con la diversidad en un aula de clases, fortalecer el compromiso social del docente. Docentes y directores necesitan ser acompañados y asistidos en este proceso de cambio y desarrollo de nuevas capacidades, que pasa por aceptar la diversidad humana como un hecho previo y positivo, una condición ineludible y necesaria de la tarea de educar. No existen mecanismos actualmente que garanticen esto y las actividades de capacitación se limitan a entregar información metodológica para el aprendizaje del lenguaje y las matemáticas.

En el caso de Venezuela, la formación del docente de educación inicial está orientada básicamente a la atención de niños entre 4 y 6 años de edad en aulas de enseñanza preescolar; no para la atención de niños de 0 a 3 años, ni para trabajar con adultos, lo que exige estrategias diferentes a las empleadas en el trabajo con niños. En relación a la educación básica, en general, el proyecto de Escuelas Bolivarianas ha desarrollado un programa de formación y sensibilización del docente para promover una práctica pedagógica abierta, reflexiva y constructiva, habiéndose creado 315 Centros Municipales de Apoyo al Docente, dotados de recursos didácticos (textos, computadoras e impresoras) de $1^{\circ}$ a $6^{\circ}$ grado.

\section{Políticas específicas de equidad}

En el caso de Bolivia, el Ministerio de educación a través de su equipo de educación especial ha trabajado varias líneas de acción: ha elaborado módulos de integración, enviados a todos los Institutos Normales Superiores para que sean incorporados; ha ofrecido formación permanente a maestros de la modalidad regular y especial, ha elaborado materiales como guías didácticas, boletines, afiches, se ha publicado la guía de adaptaciones curriculares para niños y niñas con Necesidades Educativas 
Especiales para el nivel inicial y primario. El Plan Decenal para el Desarrollo de la Mujer y la Niñez busca la expansión de la matricula, la alfabetización y una política de atención a menores en circunstancias especialmente difíciles, aunque algunas de estas iniciativas han sido discontinuadas o desfinanciadas. Últimamente se ha ampliado la obligatoriedad de la educación inicial y se ha reconocido la necesidad de su atención prioritaria. En primaria se ha creado un bono de permanencia, un incentivo para retener a los niños en la escuela otorgando un monto de dinero fijo a las familias por niño que asiste, pero es una medida aislada que no corresponde a ninguna de las líneas definidas en el nuevo proyecto de Ley de Educación, ni está acompañada por medidas a nivel curricular. No se conocen políticas nacionales o reformas recientes tendientes a estimular de manera específica las medidas y prácticas inclusivas, ni siquiera en el campo de la educación especial, que se mantienen en el plano normativo.

En Colombia, como estrategia del Ministerio de Educación Nacional para el logro de la inclusión educativa, se busca apoyar a las entidades territoriales para que, a través de las Secretarías de educación, logren avanzar en sus procesos de reorganización, asignación de docentes, coordinación de acciones necesarias para identificar y caracterizar las poblaciones vulnerables dentro y fuera del sistema; proponer orientaciones pedagógicas para elaborar y adecuar currículos; implementar, identificar y desarrollar modelos educativos flexibles; crear herramientas pedagógicas y didácticas; definir canastas educativas; formar a funcionarios, directivos docentes y docentes, entre otros.

En el Perú, la población de niños con habilidades diferentes y los niños que residen en la zona rural, han sido objeto en el último periodo de políticas de inclusión. Se han logrado avances en términos de cobertura pero, como se señaló anteriormente, el enfoque de la inclusión se asume en los hechos más como integración física a la escuela que como calidad de convivencia y adecuación de oportunidades de aprendizaje, más allá de lo que dicen las normas. De otro lado, si bien el concepto se ha abierto paso en el plano de la normatividad, falta lograr que estas normas sean conocidas y reconocidas como derecho, en primer lugar, por las propias personas excluidas y luego, por el resto de los ciudadanos. En el Perú se suele asociar la inclusión a la educación de poblaciones marginales, sea por ruralidad o discapacidad, pero no a la que se imparte a todos en el conjunto del sistema educativo nacional.

En Venezuela, es una política la transformación progresiva de los centros de Educación Preescolares estatales en Preescolares Bolivarianos, para la atención de niños entre 3 y 6 años a tiempo completo, brindando atención educativa y alimentaria. El Simoncito es un centro educativo orientado a promover el desarrollo integral del niño desde la gestación hasta los 6 años de edad, con la participación activa de la familia y la comunidad, quienes reciben atención integral (educación, alimentación, recreación y protección legal). Desde el Centro Simoncito se desarrollan dos estrategias para la atención de los niños y sus familias: la convencional, para los niños que 
asisten al Centro, y la no convencional (Simoncito Comunitario), con la salida del docente a la comunidad o al hogar de los niños no incluidos en la atención formal, desarrollando con ellos actividades formativas y de orientación. En el caso de la educación básica, se propone el proyecto de Escuelas Bolivarianas, que con jornadas escolares completas están dirigidas a asegurar la permanencia, prosecución y culminación de los períodos escolares establecidos y satisfacer, a la vez, las necesidades de alimentación y atención preventiva en salud de los estudiantes, desde una acción intersectorial y con instituciones del área de las ciencias, las artes y el deporte.

\section{Temas pendientes}

En Bolivia, si bien existen prácticas rescatables e iniciativas estatales que han marcado avances importantes en el desarrollo de una perspectiva de educación inclusiva, esto no se afronta de manera sistemática, ni aparece en términos de acciones y estrategias como prioridad del Estado. La educación inicial, por ejemplo, no debe seguir siendo entendida como una educación subsidiaria, sino como parte sustancial en la trayectoria educativa de los estudiantes. Por ello, es importante el fortalecimiento del sistema educativo público, la ampliación del sistema de educación inicial logrando calidad y mayor articulación con la primaria, con participación e igualdad de oportunidades. De otro lado, la escasa preparación de los docentes y las pocas habilidades y competencias específicas con las que éstos cuentan para desarrollar prácticas inclusivas, es un tema que merece mucha atención. La formación docente es un pilar ineludible para lograr dicha tarea y es un desafío importante mejorar las capacidades y habilidades de los docentes orientadas al fortalecimiento del trabajo con las adaptaciones curriculares. Se hace necesario prever la disposición de recursos humanos y económicos para desarrollar iniciativas que se orienten a la construcción un sistema educativo con una visión más inclusiva e integradora y que dichas políticas sean sustentables. Es necesario construir mediaciones entre las declaraciones políticas, las intencionalidades y las prácticas, de manera que sea posible operativizar y hacer realidad los principios inclusivos expresados por el Estado.

En Colombia, las políticas, estrategias e intervenciones de corte transversal para la inclusión de los niños con discapacidad, deberían apuntar a la reducción de los factores de riesgo asociados al estado de salud individual, a la situación nutricional, al aprendizaje, al desarrollo de capacidades y habilidades laborales; a los riesgos ocupacionales; así como a eventos sociales como el embarazo adolescente, la violencia, el conflicto armado, la accidentalidad y las barreras para la integración social y productiva. Desde esta perspectiva, la discapacidad se constituye en un riesgo social.

En el caso de Ecuador, la ejecución de su Plan Decenal de Educación requiere de entrada que se cumpla la política ocho: aumento del $0.5 \%$ anual de participación del sector educativo en el PIB, hasta el año 2012, o hasta alcanzar al menos el 6\% o, de lo contrario, el Plan no podrá ejecutarse. El actual gobierno se ha comprometido a ejecutar el Plan Decenal en los cuatro años de su gestión y a impulsar la aproba- 
ción de los instrumentos legales que permitirán su financiamiento. Aunque las políticas educativas por si solas no modificarán las inequidades sociales en la sociedad ecuatoriana, una educación pública de calidad sí contribuye a generar la esperanza de una vida mejor de las personas. Ecuador es un país multiétnico y pluricultural, por lo que el concepto de inclusión social necesita estar dirigido a la inclusión de los grupos indígenas. Hay que tener en cuenta que el sistema educativo ecuatoriano es excluyente y no responde a las actuales demandas sociales, se requiere un arduo trabajo para que se asuma la educación inclusiva como el cumplimiento del derecho de todas las personas a una educación de calidad, los niños con VIH, los afro descendientes, los niños de las calles, los niños trabajadores, los que tienen alguna discapacidad, entre otros grupos postergados. Además, se hacen indispensables políticas integrales de capacitación docente, estímulos, establecimiento de un sistema de evaluación de desempeño. El Plan Decenal de Educación se hace mucho más importante por cuanto contempla estos aspectos de mejoramiento profesional.

En el Perú, como en otros países de América Latina, pese a las políticas y los esfuerzos del Estado, la escuela no ha logrado brindar igualdad de oportunidades a los que están dentro; sólo un grupo reducido se beneficia con la posibilidad de continuar con su educación y la escuela sigue siendo un instrumento para mantener las diferencias, para mantener a grupos excluidos de esta posibilidad. Los niveles socioeconómicos siguen determinando el nivel de los logros educativos que los estudiantes consiguen. Los bajos niveles de educación permanecen asociados a la pobreza total o extrema, a las zonas rurales y al nivel educativo de los padres. La educación en el área rural es la menos favorecida como se ve en los índices de ingreso, permanencia, egreso y logro de aprendizajes. Hay algunos progresos en relación a la situación que se presentaba a inicios de los 90, pero la educación sigue sin cumplir una función en pro de la convivencia y la integración entre los peruanos.

En Venezuela, el alto costo de los servicios ofrecidos a la población preescolar por la diversidad de servicios, el alto número de recursos humanos necesarios para su implementación, así como la especial estructura requerida para atender niños preescolares y maternales, demanda un seguimiento y una evaluación permanente de su efectividad y eficiencia en el logro de sus objetivos, en particular, del éxito de los procesos formativos y compensatorios, que aseguren la no repitencia en el primer grado y la esperada prosecución escolar de los niños atendidos, así como la esperada integración de los niños excluidos del sistema preescolar y de aquellos menores de 3 años y sus familias en mayores riesgos sociales y económicos. Por sus características se aconseja concentrarlo en las poblaciones de menores recursos y revisar su aplicabilidad para el caso de la población rural e indígena. De otro lado, la meta de convertir todas las escuelas básicas en Bolivarianas no ha sido lograda. La transformación de las escuelas regulares en Bolivarianas y la construcción de nuevas escuelas, han encontrado dificultades administrativas y problemas de gestión. Convertir el medio turno escolar en turno completo conlleva negociaciones con los gremios docentes y la 
aplicación de una logística administrativa que en muchos casos no se ha cumplido. El servicio de alimentación exige también una eficiente coordinación interinstitucional, difícil de lograr desde una gestión centralizada.

\section{Conclusiones generales: los cuatro desafíos de la inclusión en la Región}

\section{Cobertura}

Los múltiples sentidos de la inclusión se hacen patentes en el análisis de las políticas educativas en los países de la Región Andina, como presencia o ausencia, como problema o posibilidad. La inclusión como acceso a la educación es la que encuentra mayor visibilidad en las acciones de gobierno. Bolivia ha llegado al 2001 con una cobertura bruta de matrícula incrementada en todos los niveles: en primaria, con tasas superiores al $100 \%$, lo que significa que está atendiendo a una cantidad mayor a la población objetivo (niños de 6 a 13 años), en el nivel inicial, con un crecimiento de $59,9 \%$ y de $34,5 \%$ en el nivel secundario. En el caso de Colombia, las políticas de inclusión se han hecho extensivas a los desvinculados de las organizaciones al margen de la ley y a la población desplazada por sus acciones, además a la población rural, a personas con necesidades educativas especiales e incluso a aquellas con talentos excepcionales tempranamente detectados. En Ecuador, la tasa de escolaridad de educación básica entre 1999 y 2006 subió de $87.80 \%$ a $90.80 \%$, y en el caso de la educación rural, subió de $87.90 \%$ a $94.36 \%$. En el caso de Perú, en las dos últimas décadas la tasa de cobertura aumentó de manera importante en todos los niveles educativos, atendiéndose al $53,6 \%$ de la población de 3 a 5 años, al $91,9 \%$ de la población de 6 a 11 años, y al 72,8\% de la población de 12 a 16 años de edad. En el caso de Venezuela, el presupuesto de educación se ha aumentado a más del 7\% del PIB y se ha priorizado la cobertura educativa a los grupos más pobres, la población infantil y juvenil de zonas rurales e indígena y de fronteras, y los niños y jóvenes excluidos del sistema educativo formal. Bajo ese impulso se han alcanzado niveles de universalización en la educación de niños de 5 y 6 años y se ha llegado a una tasa neta de $89,6 \%$ en la matrícula de educación básica.

Esto no quiere decir que no exista a este nivel una agenda pendiente. Los informes registran dificultades y sesgos aún por resolver. Por ejemplo, los modelos educativos que se eligen para extender la atención educativa no siempre poseen la necesaria pertinencia sociocultural, estrategias efectivas para el desarrollo de capacidades del personal destinado a operar con ellos, el financiamiento asegurado para lograr sus metas en periodos razonables, ni estrategias definidas para avanzar en medio de previsibles obstáculos y una alta diversidad de contextos. Pero también hay problemas en relación con los criterios de priorización de la población desatendida, constatándose el enorme déficit de servicios actualmente existente para los niños menores de 5 años y para la población adolescente en general, más aún 
cuando se trata de sectores que ven agravada su exclusión cuando se les añade otras desventajas como la pobreza, la ruralidad, el desplazamiento, la violencia armada o la discapacidad, entre otras.

\section{Condiciones materiales}

La que ha tenido presencia notoria, así mismo, en la acción de los Estados, pese a sus deficiencias e insuficiencias, es la inclusión como acceso equitativo a condiciones materiales básicas para el aprendizaje. En el caso de Bolivia, se encuentran iniciativas estatales que buscan ampliar la cobertura y la permanencia a través de bonos de asistencia integral alimentaria y de atención en salud a la primera infancia, derivadas de políticas de reducción de la pobreza. Así mismo, programas como Wawa Wasi (9.000 beneficiarios) y Kallpa Wawa (16.000 beneficiarios), dirigidos a poblaciones rurales e indígenas muy pobres, dan atención a niños de 3 a 5 años a nivel de su preparación para la escuela, su protección social, su salud y seguridad alimentaria. También, el Programa Nacional de Atención al Niño y a la Niña menor de 6 años (PAN), cuyo objetivo es brindar atención integral a niños menores de 6 años de los sectores más empobrecidos, a través de centros infantiles integrales familiares y comunitarios. Colombia viene desarrollando una política de inclusión basada en normas y planes de acción dirigidos a crear condiciones apropiadas y orientar a las diferentes entidades gubernamentales nacionales, territoriales y locales, instituciones, centros educativos y personas, para conseguir que la mayor parte de los niños, niñas y jóvenes de las etnias accedan, permanezcan y se promocionen dentro del sistema educativo, según sus condiciones particulares. Ecuador, por su parte, como resultado de muchos esfuerzos de las autoridades centrales y sectoriales, ha disminuido los niveles de desnutrición infantil en los últimos años e incrementado el acceso a servicios de la población en general, aumentando así mismo el nivel de dotación de insumos a los centros educativos públicos. Perú, a su vez, ha desplegado más de 4.600 hogares comunitarios en todo el país, dirigidos a niños menores de 3 años, habiendo logrado disminuir los niveles de desnutrición de sus usuarios. El Programa Nacional de Apoyo Directo a los más Pobres, denominado Juntos, atendió a más de 300.000 familias de 623 distritos más pobres del país, beneficiándolas con un incentivo económico a cambio de que las madres hagan control de su embarazo, lleven a sus hijos a los puestos de salud, los matriculen y mantengan en la escuela, y asistan a los programas de nutrición. Venezuela se ha empeñado en la transformación de las escuelas regulares en Bolivarianas, lo que ha implicado la conversión del medio turno escolar en turno completo y la implementación del servicio de alimentación estudiantil. Así mismo, está impulsando el programa Simoncito, un centro educativo para niños de 0 a 6 años de edad, con la participación activa de la familia y la comunidad, brindándoles a tiempo completo atención integral: educación, alimentación, recreación y protección legal, en la perspectiva de convertirlo en un canal de llegada de las políticas sociales compensatorias. 
A pesar de estos progresos, hay también en este ámbito una agenda pendiente. Los porcentajes de población excluida que son atendidos por diversas modalidades de apoyo -subsidios directos, atención de salud, insumos materiales, apoyo nutricional, protección legal- siguen siendo minoritarios y subsisten problemas de coordinación y articulación entre estos programas. En ese sentido, las desventajas de inicio que presentan los estudiantes que provienen de estas poblaciones críticas, juegan en contra de sus posibilidades de éxito escolar en una gran mayoría y las brechas de equipamiento básico entre las escuelas urbanas y rurales sigue siendo clamoroso. En varios casos, además, las políticas presupuestales tampoco hacen distingo de las desigualdades sociales y educativas, persistiendo en asignaciones homogéneas de recursos a todos los sectores y manteniendo un promedio uniforme de inversión por alumno.

\section{Logros}

La inclusión que presenta mayores dificultades en todos los casos, pese a ser la más directamente relacionada al currículo, es la inclusión como acceso a logros educativos de calidad. En todos los países el rendimiento escolar en áreas como el dominio del lenguaje o las matemáticas son bastante malos, en especial los del sistema público de educación y, sobre todo, los de la escuela rural. Más allá de los avances en relación a la cobertura y los esfuerzos por la permanencia escolar, Bolivia reconoce tener un sistema educativo débil, con claros rasgos de ineficiencia y exclusión, rígido en términos de mentalidad y prácticas educativas, siendo predominante una visión exclusivista, competitiva y homogenizadora de la enseñanza. No se evidencia que, aún con los planteamientos de inclusión presentes en el currículo inicial y básico, los procesos de cambio curricular estén propiciando prácticas inclusivas en el aula ni una mayor apertura a considerar la diversidad de habilidades y capacidades de los estudiantes y la brecha rural urbana sigue siendo de consideración. En Colombia se reconoce que se ha logrado avanzar en cobertura, pero sin garantizar la permanencia en el sistema de todos los estudiantes ni tampoco una educación básica de calidad, haciéndose patente la dificultad para traducir las normas sobre la inclusión en prácticas pedagógicas realmente inclusivas. En el caso de la inclusión de personas con necesidades educativas especiales, preocupa que no se haya garantizado en los docentes las capacidades pedagógicas necesarias para trabajar con ellos apropiadamente. En Ecuador, pese a su Reforma curricular, todavía tienen vigencia los esquemas pedagógicos tradicionales, basados en el memorismo, el maltrato, el autoritarismo y la discriminación en las escuelas, textos y material didáctico poco pertinente y desactualizado. En el caso de Perú, más allá de las bondades en el enfoque inclusivo del currículo de educación básica, se detectan dificultades en su implementación: maestros insuficientemente capacitados y no convencidos de la nueva orientación del currículo, sin acompañamiento profesional a su desempeño en las aulas, contratación tardía de maestros en muchas escuelas, lo que obliga después a 
abreviar el currículo y a desarrollarlo con premuras; no difusión de los cambios a las familias y la comunidad para orientar la demanda hacia nuevos criterios de calidad, evaluaciones periódicas del rendimiento que no son seguidas de medidas correctivas, resultando que los grupos sociales antes excluidos de la educación, pese a tener ya acceso a la escuela, siguen sin acceso a aprendizajes de calidad. En Venezuela hay preocupación por la no evaluación de los nuevos modelos propuestos para la educación inicial y básica, a fin de comprobar su eficacia en su contribución a disminuir la repitencia, deserción y exclusión. Persisten, por ejemplo, las deficiencias en la educación de los niños que ingresan al primer grado, pues la no adecuación de la enseñanza a sus estilos de aprendizaje no garantiza el desarrollo de las habilidades que requieren para continuar su trayectoria escolar. Persisten, así mismo, los problemas en el desempeño de los docentes, que no se muestran hábiles en el manejo de una pedagogía basada en la acción y la construcción de conocimientos, ni encuentran la manera de asegurar una transición exitosa de sus estudiantes de un ciclo a otro de la escuela básica.

La agenda pendiente en este ámbito aparece más evidente. Es aquí, donde entran en juego de una manera más directa las subjetividades de los actores, sus sentidos comunes, sus hábitos y creencias, así como las culturas de las instituciones a las que pertenecen, donde menos capacidad de respuesta parecen tener los Estados, habituados a manejarse desde una lógica jerárquica y normativa. Aprender a enseñar con eficacia a un aula diversa y afectada por las desigualdades sociales, rescatando potencialidades y construyendo saberes sobre los que ya se traen, supone un giro pedagógico de tal magnitud que cuestiona las bases mismas de la identidad y la formación profesional de los docentes. No es esperable que, en este contexto de desafío, los maestros puedan transitar hacia un nuevo rol sin apoyos calificados y sostenibles, ni mecanismos institucionalizados de soporte pedagógico y socio emocional. Es en este terreno donde la dotación de insumos, la instrumentación didáctica y las nuevas reglamentaciones, medios privilegiados por las políticas dirigidas a acortar brechas de equidad educativa, tocan sus límites. La inclusión de todos los sectores excluidos en el acceso a logros de igual calidad representa un desafío de mayor talla, que requiere actores convencidos y realmente habilitados para desempeñarse con apertura y flexibilidad en escenarios educativos muy heterogéneos, socialmente desiguales y, por eso mismo, más exigentes.

\section{Integración}

No obstante, la que registra los mayores problemas y desafíos es la inclusión educativa como inclusión social y cultural de todos los grupos sociales, una inclusión con respeto a sus identidades y en una perspectiva de integración social. En Bolivia se señala que el Estado no ha logrado ser constructor de ciudadanía ni la educación posibilitar la construcción de una identidad nacional, siendo un país con una composición social muy compleja y culturalmente diversa, donde han convivido 
por siglos estructuras sociales superpuestas. En ese contexto se considera difícil que algunas medidas de carácter curricular pueden generar en la escuela un contexto más favorable para una educación inclusiva, abstraída del sustrato ético, político e ideológico que conlleva el concepto de inclusión educativa. En Colombia se señala que, además de políticas más eficaces para la inclusión, urge cambiar la mirada de la sociedad frente al sector más pobre y excluido de la población, para que no sean estigmatizados en sus desventajas ni desistan de culminar la escolaridad básica, para que sean reconocidos como sujetos con iguales derechos, lo que significa un reto cultural y político para los colombianos, pero también a nivel de práctica pedagógica. En el caso de Ecuador, se señala que el racismo es una de las formas de exclusión y maltrato que todavía se observa en la formación de muchos niños indígenas y afro americanos, siendo un hecho que aún estando en escuelas bilingües tienen menos probabilidades de aprobar el grado que los niños no indígenas que estudian en las escuelas denominadas hispanas. En el caso de Perú, se afirma que la exclusión nunca ha sido sólo un problema de no acceso a la educación formal, pues cuando grupos sociales antes marginados logran ser incluidos terminan siendo subvalorados, estigmatizados y tratados como marginales, tendiendo a ser expulsados por el sistema; y no se constata que la educación esté cumpliendo una función a favor de la convivencia, la integración y la cohesión social entre los peruanos. En Venezuela se señala que la inclusión educativa no será posible mientras persista en el país la exclusión y la polarización política, en la medida que es una barrera para el diálogo y un factor que acrecienta la división política, social y económica del país.

La agenda pendiente de la inclusión como integración social es más compleja, pero necesita ser afrontada. Las posibilidades de progreso en los estándares de calidad pedagógica del desempeño docente, su misma especialización en contextos de desigualdad y exclusión, puede encontrar una barrera insalvable en el prejuicio y la discriminación instalada en la cultura escolar, tanto como en la falta de convicción de los decisores respecto de la gravedad de este problema y de la necesidad ineludible de abordarlo desde las políticas. Que en las escuelas todos los actores involucrados se sientan o no iguales en esencia, es un problema de valoración y es un problema de confianza, y no surgirá por prescripción sino por experiencia. Estas experiencias de cohesión interna en función a objetivos comunes, basadas en la confianza y por lo tanto en la cooperación y la complementariedad, necesitan ser construidas desde un modelo de gestión, en general, opuesto al modelo administrativo que prevalece en las instituciones escolares. Como se ha dicho ya, en este campo también hay lecciones de la experiencia que deben ser capitalizadas por las políticas oficiales.

\section{Reflexiones finales}

La mayoría de la población de los países de la Región Andina vive en la pobreza. Los informes nacionales no dejan lugar a dudas: no menos de la mitad y más de las dos terceras partes de su sociedad, dependiendo de las zonas, del idioma que 
hablen y de su lejanía de las ciudades principales, son pobres, siendo los niños los más seriamente afectados y los menos atendidos en todos los casos. Es a esa población justamente a quien la educación y los demás servicios del Estado llegan menos, llegan mal y, por si fuera poco, llegan desajustados de sus necesidades y ajenos a su comprensión de la vida. En ese contexto se plantea la pregunta por el carácter inclusivo de los currículos escolares de nuestros países.

Un currículo puede estar formulado de manera no inclusiva, reflejando estereotipos discriminadores y acentuando su tradicional etnocentrismo, lo cual haría difícil cualquier esfuerzo de educación inclusiva desde la educación formal. No parece ser, estrictamente, el caso de los países andinos, donde sus currículos se han ido transformando y flexibilizando progresivamente en los últimos 20 años para dar cabida a la diversidad social, aún cuando sigan siendo débiles, escasos y desiguales los resultados en el esfuerzo por transitar de la multiculturalidad a la interculturalidad.

El problema principal parece estar en tres frentes: de un lado, en la implementación del currículo; es decir, en las estrategias empleadas para que cobre vida y tenga impacto real en las aulas, en las complejas y muy diversas condiciones en que debe enseñarse; de otro lado, en la pertinencia de sus contenidos respecto de lo que nuestras sociedades necesitan para integrarse y cohesionarse alrededor de objetivos comunes de desarrollo humano e igualdad social; adicionalmente, en los otros procesos de reforma que acompañan (o no) las políticas curriculares implementadas, haciendo posible y/o reforzando y complementando su impacto.

En primer lugar, lo que se lee en los informes nacionales es que las políticas de implementación no parecen tener mucho éxito en su lucha contra resistencias y barreras en la práctica docente, cuyas premisas pedagógicas no suelen coincidir con las de los currículos, y en las propias instituciones escolares, que no ceden fácilmente en su manera de organizarse y gestionarse a esquemas más flexibles y ajustados a las características de la población que atienden. No parece ser un problema de plazos, argumento con el que se suele justificar la falta de resultados de las políticas educativas, sino de enfoque: surge la pregunta de hasta qué punto las políticas de implementación se agotan en las prescripciones y no avanzan en la difícil pero indispensable tarea de construir las condiciones objetivas y subjetivas para allanar el camino y que sus objetivos se cumplan sin atenuantes. Desde esta perspectiva, se ha señalado la apremiante necesidad no sólo de formar mejor a los docentes -cuestión generalmente reducida a aspectos técnicos muy específicos de la enseñanza como si el problema fuese sólo y principalmente operativo- sino de discutir los enfoques y premisas en que descansan sus prácticas habituales, para construir con ellos nuevos acuerdos sobre los nuevos sentidos de los aprendizajes hoy demandados por el currículo, sobre sus enormes implicancias para la enseñanza y, por lo tanto, para el significado mismo de la profesión docente y para la misión institucional de la escuela.

En segundo lugar, aún si el problema anterior no existiese o no fuese tan grave, todavía sería posible preguntarse si lo que nuestros niños parecen estar aprendiendo 
bien, incluso en su idioma materno y en contacto continuo con sus propios referentes culturales, es acaso lo que necesitan saber pensar y saber hacer para no reproducir una sociedad dividida y profundamente desigual. Los informes distinguen la inclusión en relación con las oportunidades de acceder equitativamente a aprendizajes de calidad, de la inclusión social; es decir, del respeto profundo por las identidades de los niños, de una genuina valoración de la diversidad de rasgos y perspectivas al interior de un aula, de un auténtico diálogo intercultural, que suponga intercambios y complementariedades en todos los planos de la relación humana.

En tercer lugar, todos los informes dan cuenta de otros procesos de reforma implementados en paralelo, de cuya fortuna depende también la posibilidad de avanzar en materia de inclusión en las aulas. Un aspecto crítico, por ejemplo, tiene que ver con la política docente, más allá de los sin duda necesarios programas de formación. Se ha señalado la paradoja de encargar la implementación de políticas curriculares inclusivas a las propias víctimas de la exclusión educativa, en cuya trayectoria formativa no figuran antecedentes relevantes de inclusión. Todos sabemos de las enormes precariedades y obsolescencias de su formación profesional inicial, de la pobreza material de sus familias y de la mala educación básica de la que provienen en la mayor parte de casos, efectuada por lo general en el segmento más excluido y excluyente del sistema público.

En varios casos hay procesos en ciernes o en marcha para instalar mecanismos de evaluación del desempeño docente y nuevas reglas de juego para el ejercicio de la profesión. Siendo todo esto necesario, sin embargo, hacen falta políticas más enérgicas y efectivas de desarrollo profesional, que sirvan justamente para suplir estas enormes desventajas y avanzar en los niveles de profesionalización de la docencia, proceso que no se va a desatar ni a alimentar con medidas de control. Los docentes que deben hacerse cargo de las escuelas más pobres del sistema y de la población más excluida de la sociedad, deben tener una preparación más específica, así como herramientas y condiciones especiales para el ejercicio de su labor. La evaluación docente debe servir fundamentalmente para medir los progresos en esa dirección.

Por último, no se puede perder de vista que los sistemas educativos y los currículos únicos nacieron históricamente como entidades profundamente etnocéntricas dirigidas a uniformizar identidades, donde lo diverso era visto como dificultad y era objeto de menosprecio e imposición cultural. Esa es la herencia histórica que se tiene que enfrentar y vencer para hacer posible la inclusión en la educación de los niños, una inclusión entendida como integración basada en el respeto a las diferencias, no como homogenización. En el fondo se trata de redefinir los términos del contrato social implícito que dio origen a estos sistemas, pésimos remedos además de los sistemas europeos, tanto como su diseño mismo. No es posible construir una escuela inclusiva al interior de estas mismas estructuras, hechas para excluir los insumos defectuosos (léase los diferentes) y procesar al resto de manera uniforme, como en una línea de montaje industrial, sin que la poderosa inercia del sistema termine a 
la larga por normalizarla, arrastrándola de regreso a un esquema básico de funcionamiento auto centrado y excluyente, con todas sus herramientas y mecanismos inclusivos adentro.

Es por eso que las normas no bastan. El desafío es identificar y enfrentar estas barreras y resistencias en cada caso, construir las condiciones y convicciones en los actores y en las instituciones para que las normas sobre inclusividad y el currículo se traduzcan en prácticas inclusivas efectivas a nivel educativo y social. Y esto es responsabilidad principal de los Estados, en tanto garantes de la igualdad de oportunidades, y esto es, además, lo que debe dar contenido a las políticas públicas sobre inclusión educativa, en el marco del currículo y los aprendizajes escolares.

De todos modos, en materia de formulación curricular es todavía amplio el terreno por recorrer. Se han señalado los progresos en este terreno en toda la Región Andina y, sin embargo, todavía requieren convertirse en instrumentos más legibles para los maestros y las familias, en expresiones más contundentes de la diversidad geográfica, económica, social y cultural de nuestros países, en fuentes mucho más claras de demandas a las prácticas pedagógicas de los docentes, en símbolos de consensos sociales muy amplios respecto de lo que cada país busca para sus ciudadanos en un horizonte de desarrollo humano. Antes se pensaba que la llave para el cambio de la educación era la reforma curricular y que todo lo demás caería por su propio peso. Hoy se sabe que no es así y que necesita converger con otras variables, medidas y políticas para aportar a los objetivos de calidad con equidad y, específicamente, de inclusión educativa en el tercer y cuarto nivel de desafío que se ha señalado antes. Pero sin volver a reformarse, entrando de nuevo en un ciclo interminable de recambios como un mito griego, pueden seguir mejorando, especificándose, universalizándose y a la vez interculturalizándose, emparentándose de un modo más ideológico y no sólo metodológico, con un indispensable movimiento de reforma de la pedagogía escolar y de la gestión de la escuela.

Más allá de la cobertura y la universalización, tenemos el imperativo de lograr calidad para todos; equidad en los logros y resultados; pertinencia basada en un real reconocimiento del otro como sujeto de derecho. Más allá de normas y prescripciones, tenemos el imperativo de construir condiciones objetivas y subjetivas, materiales y simbólicas, para favorecer que los currículos tengan un real impacto en las aulas y en los sujetos. Más allá de los discursos, tenemos el imperativo de cambiar mentalidades y prácticas para no reproducir sociedades profundamente divididas y desiguales para respetar las identidades de los niños, para valorar genuinamente la diversidad de rasgos y perspectivas en el aula, para respetar las diferencias. Más allá de recibir y retener a todos y de posibilitarles concluir la escolaridad, tenemos el imperativo de recibir a cada uno con hospitalidad: la calidad de nuestras sociedades vista a través del modo en que se recibe/recibimos a los que llegan. Tenemos, así, el imperativo de mejorar las condiciones para recibir mejor a los que seguirán llegando. Ese es hoy nuestro reto. 


\section{Bibliografía}

Aguerrondo, I. (2007). Exclusióninclusión. Manuscrito no publicado. Disponible en http://www.udesa.edu. ar/files/EscEdu/Inclusi\%C3\%B3n \%20 Educativa $/ 41 \% 20$ In $\%$ C3\%A9s $\% 20$ Aguerrondo\%20_Argentina_.pdf

Calvo, G. (2007). Colombia. La dificultad para concretar las normas en inclusión educativa. Ginebra: UNESCO, Oficina Internacional de Educación.

Guerrero, L. y Rivas, K. (2007). Currículo e inclusión en el nivel inicial y el primer ciclo de la primaria: Visiones, estrategias, experiencias y buenas prácticas en la Región Andina. El caso de Perú. Ginebra: UNESCO, Oficina Internacional de Educación.

JiMÉNEZ, C. (2007). Currículo e inclusión en el nivel inicial y el primer ciclo de la primaria: Visiones, estrategias, experiencias y buenas prácticas en la Región Andina. El caso de Ecuador. Ginebra: UNESCO, Oficina Internacional de Educación.

Tenti, E. (2007). Dimensiones de la exclusión educativa y las políticas de inclusión (borrador para la discusión, agosto de 2007). Ponencia presentada en el Taller Internacional sobre Inclusión Educativa-América Latina, Regiones Cono Sur y Andina, Buenos Aires, Argentina. Disponible en www.ibe.unesco. org/Spanish/cops/workshops/Argentina/ Inclusion_exclusion_SPA.pdf

TERÁN, M. (2007). Currículo e inclusión en el nivel inicial y el primer ciclo de la primaria: Visiones, estrategias, experiencias y buenas prácticas en la Región Andina. El caso de Venezuela. Ginebra: UNESCO, Oficina Internacional de Educación.

UnesCo (2005). Guidelines for inclusion: Ensuring access to education for all. París: UNESCO. Disponible en http://unesdoc.unesco.org/ images/0014/001402/140224e.pdf

UNESCO-OIE (2007). Brief conceptual note about inclusive education. Manuscrito no publicado.

WeIsE, C. (2007). Currículo e inclusión en el nivel inicial y el primer ciclo de la primaria: Visiones, estrategias, experiencias y buenas prácticas en la Región Andina. El caso de Bolivia. Ginebra: UNESCO, Oficina Internacional de Educación. 